\title{
Multiscale Model for the Templated Synthesis of Mesoporous Silica: The Essential Role of Silica Oligomers
}

\author{
Germán Pérez-Sánchez, ${ }^{\dagger, \ddagger}{ }^{\S}$ Szu-Chia Chien, ${ }^{\|, \perp}{ }^{\prime}$ José R. B. Gomes, ${ }^{\dagger}$ M. Natália D. S. Cordeiro,
} Scott M. Auerbach, ${ }^{\perp, \#}$ Peter A. Monson, ${ }^{\perp}$ and Miguel Jorge ${ }^{*}, \diamond, \S$

${ }^{\dagger}$ CICECO, Departamento de Química, Universidade de Aveiro, Campus Universitário de Santiago, 3810193 Aveiro, Portugal

${ }^{\ddagger}$ REQUIMTE, Department of Chemistry and Biochemistry, Faculty of Sciences, University of Porto, Rua Campo Alegre 687, 4169007 Porto, Portugal

${ }^{\S}$ Laboratory of Separation and Reaction Engineering (LSRE), Associate Laboratory LSRE/LCM, Faculdade de Engenharia, Universidade do Porto, Rua Dr. Roberto Frias, 4200465 Porto, Portugal

${ }^{\perp}$ Department of Chemical Engineering and ${ }^{\#}$ Department of Chemistry, University of Massachusetts, 686 North Pleasant Street, Amherst, Massachusetts 01003 9303, United States

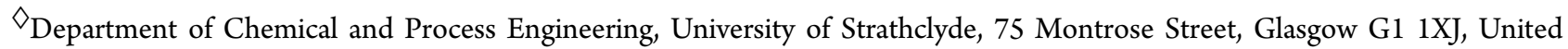
Kingdom

Supporting Information

ABSTRACT: A detailed theoretical understanding of the synthesis mechanism of periodic mesoporous silica has not yet been achieved. We present results of a multiscale simulation strategy that, for the first time, describes the molecular level processes behind the formation of silica/surfactant mesophases in the synthesis of templated MCM 41 materials. The parameters of a new coarse grained explicit solvent model for the synthesis solution are calibrated with reference to a detailed atomistic model, which itself is based on quantum mechanical

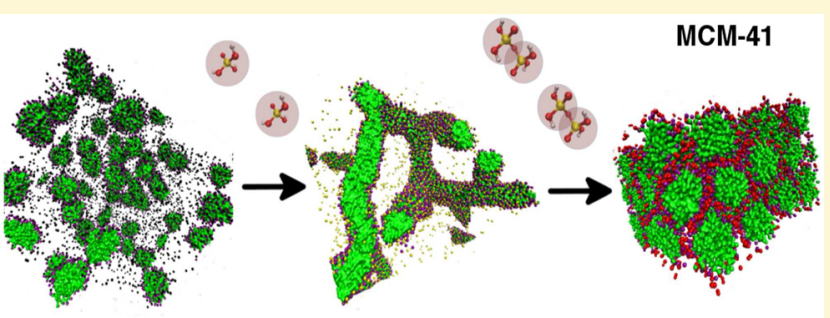
calculations. This approach allows us to reach the necessary time and length scales to explicitly simulate the spontaneous formation of mesophase structures while maintaining a level of realism that allows for direct comparison with experimental systems. Our model shows that silica oligomers are a necessary component in the formation of hexagonal liquid crystals from low concentration surfactant solutions. Because they are multiply charged, silica oligomers are able to bridge adjacent micelles, thus allowing them to overcome their mutual repulsion and form aggregates. This leads the system to phase separate into a dilute solution and a silica/surfactant rich mesophase, which leads to MCM 41 formation. Before extensive silica condensation takes place, the mesophase structure can be controlled by manipulation of the synthesis conditions. Our modeling results are in close agreement with experimental observations and strongly support a cooperative mechanism for synthesis of this class of materials. This work paves the way for tailored design of nanoporous materials using computational models.

\section{INTRODUCTION}

Templated synthesis is a key concept in current efforts to design nanoporous solids with tailored properties to suit particular applications and is relevant to a wide range of materials, including zeolites, periodic mesoporous silicas (PMS), porous carbons, and metal-organic frameworks. ${ }^{1}$ Templated materials come in a rich variety of structures and offer great control over the porous network properties, thus opening up the possibility for true computer based design, whereby a particular set of synthesis conditions is selected through virtual screening to yield a material with ideal properties for the target application. Computational design of materials would bring tremendous savings to current processes by minimizing expensive and time consuming laboratory experiments and would also provide an avenue for the discovery of completely new materials. Despite many advances, a complete theoretical understanding of the role of the template molecules (or supramolecular arrangements) in the synthesis process as well as of their interactions with the building blocks of the porous scaffold has not yet been achieved. ${ }^{2,3}$ This lack of understanding is preventing us from achieving true computer based design, since the crucial link between synthesis conditions and material properties remains elusive. In this paper, we take an important step in this direction by presenting a computational model based on a multiscale simulation strategy that is able to describe in detail the templating mechanism behind the synthesis of PMS materials.

Since the 1990s periodic mesoporous silicas have attracted great attention, stemming mainly from their wide range of applications, such as shape selective catalysis, gas separation by

Received: January 28, 2016

Revised: April 4, 2016

Published: April 4, 2016 
adsorption, drug delivery, as well as optical and electronic devices. $^{4-8}$ The archetypal PMS material is MCM 41, discovered by Mobil researchers more than two decades ago. ${ }^{9,10}$ It exhibits a highly ordered hexagonal arrangement of nearly cylindrical pores with sizes of a few tens of Angstroms, but the molecular structure of the pore walls is amorphous. The final properties of the material, pore size distribution, wall thickness, etc., are strongly dependent on the synthesis conditions, concentration, charge and length of the organic template, inorganic/organic ratios, $\mathrm{pH}$, and temperature. In particular, it is now well established that the topology of the porous network mirrors the structure of silica-surfactant hexagonal liquid crystals (HLC) formed early in the synthesis process. HLC formation involves several complex processes, such as surfactant self assembly, silica condensation, nucleation, and phase transitions, which can all take place simultaneously. This makes it a very challenging system to study from both experimental and modeling points of view, and as a consequence a full mechanistic description of MCM 41 formation is not yet a reality.

The original and most common experimental pathway for MCM 41 synthesis starts from a solution of cationic surfactant molecules (most often cetyltrimethylammonium bromide, or $\mathrm{CTAB}$ ) in water to which a silica source is added (most often tetraethylorthosilicate, or TEOS) under high $\mathrm{pH}$ conditions. Very early in the synthesis a silica-surfactant HLC mesophase forms, with cylindrical surfactant micelles surrounded by silicates (and sometimes other ions). With time and/or heating, the silica scaffold condenses within the mesostructure, which is then removed by calcination, producing the hexagonally ordered silica pore network. Crucially, these silica-surfactant hexagonal phases are formed even at very low surfactant concentrations, where one would expect isolated micelles to be the dominant phase. ${ }^{11}$ The uncertainty regarding the mechanism of formation of the HLC was reflected in the original mechanistic diagram of Beck et al., ${ }^{9}$ where multiple alternative pathways were suggested as leading to MCM 41 formation.

A large number of experimental studies using different techniques have tried to further elucidate this mechanism (for a more detailed discussion, the reader is directed to compre hensive reviews $s^{4,12-14}$ and to the introduction sections of our previous papers ${ }^{15,16}$ ), but important questions remain unan swered. For instance, evidence is mounting in support of a cooperative templating mechanism (CTM) whereby silicates play an active role in directing the formation of the mesostructure, ${ }^{16,17}$ but it is not clear whether an initially proposed liquid crystal templating mechanism, ${ }^{9}$ where silica is mainly a spectator during the initial stages, remains a viable alternative. Two conflicting pathways have also been proposed within the CTM: one in which silica preferentially interacts with free surfactant monomers while micelles act as reservoirs for the separately growing mesophase ${ }^{18,19}$ and another in which silicates replace counterions at micelle surfaces inducing shape transitions and aggregation of micelles. ${ }^{20-24}$ In the former case the pathway from silica-surfactant molecular clusters to ordered mesoscale phases remains largely unclear. Even in the latter case the pathway leading from silica coated spherical micelles to silica-surfactant HLC, either through sphere to rod transitions followed by rod aggregation ${ }^{20-22}$ or through spherical micelle aggregation followed by internal shape rearrangements, $^{23,24}$ is also ambiguous. In this article, we apply for the first time multiscale molecular modeling techniques to elucidate the ingredients necessary for mesoscale ordering in these systems.

To complement experiments and help answer these questions, several computational modeling studies have been reported over the past decade or so. ${ }^{25}$ One particular direction, pioneered by Siperstein and Gubbins, ${ }^{26,27}$ is to apply highly coarse grained lattice models that are designed to capture the basic physics underlying the MCM 41 synthesis process. Using a simple chainlike surfactant model ${ }^{28}$ on a simple cubic lattice and a set of predefined effective interactions between silicate and surfactant species, those authors were able to show that under certain conditions the system phase separates into a dilute solution and a concentrated HLC phase. In the silica/ surfactant rich phase they found hexagonal or lamellar mesostructures depending on the silica/surfactant ratio, in qualitative agreement with experimental results. ${ }^{29}$ This model was later extended by Jin et al., ${ }^{16}$ who used a body centered cubic lattice to more realistically represent silica connectivity, explicitly account for silica polymerization reactions, and implicitly consider the effect of $\mathrm{pH}$ on the process. When their simulations were run in two steps, the first representing the silica-surfactant HLC formation and the second allowing for extensive silica condensation, the model equilibrated to form structures that very closely resembled MCM 41 . Conversely, if extensive silica condensation was allowed to proceed from the very start, the system became locked in a highly disordered state resembling an amorphous silica gel. This confirms that the HLC mesophase is formed rather early in the synthesis, before a highly cross linked silica network is able to build up. The authors also observed a reversible hexagonal to lamellar transition upon changing the temperature of the system.

Although we have learned a lot about the PMS synthesis mechanism from lattice models, they are nevertheless based on rather drastic a priori assumptions about the interactions between different species and the possible structures that may form, which makes it difficult to establish a direct connection with experimental systems and to obtain quantitative structural predictions. Detailed atomistic models, on the other hand, generally do not suffer from this limitation. In an effort to shed light on the initial stages of MCM 41 synthesis, Jorge and co workers $^{15,30}$ extended an existing model for silicates ${ }^{31}$ to include anionic species ${ }^{32}$ and combined it with a model for $\mathrm{CTAB}$ in aqueous solution. Their simulations showed that silicates replace bromide ions at the micelle surface and thus promote micellar growth, ${ }^{30}$ in agreement with the experimental observations of Baute et al. ${ }^{33}$ They also observed that more highly condensed anionic oligomers tend to adsorb to more than one micelle at the same time, suggesting that they might play a role in promoting micellar aggregation. Unfortunately, detailed atomistic simulations are limited to small system sizes and short times, so later steps of the mechanism including mesostructure formation could not be probed.

The main aim of the present paper is to bridge the gap between the two modeling approaches described above by way of a multiscale simulation strategy. The idea is to progressively move from higher levels of theory, where computer time imposes severe limitations, to more coarse grained representa tions that allow us to access the time and length scales that are relevant to MCM 41 formation. Crucially, this allows us to maintain a close correspondence between our coarse grained model and realistic experimental systems, thus providing a much more accurate molecular level description of the 
synthesis process. A first step in this direction has already been taken, where we proposed a new model for silicic acid monomers $^{34}$ within the MARTINI coarse grained ap proach $^{35,36}$ that was benchmarked against structural properties from atomistic simulations. ${ }^{30}$ The model was able to show in detail how the presence of silica monomers promotes sphere to rod transitions in $\mathrm{CTAB}$ solutions, which take place through successive micelle fusion events. ${ }^{34}$ In the present paper, we extend this model to describe the behavior of silica oligomers in surfactant solutions and apply it to elucidate the formation of the HLC mesostructure during the early stages of MCM 41 synthesis.

Our experimental benchmark for this study is the work of Firouzi et al., ${ }^{11}$ which remains one of the most detailed experimental studies of MCM 41 synthesis starting from a low surfactant concentration solution. The authors made use of nuclear magnetic resonance spectroscopy (NMR), X ray scattering, and polarized optical microscopy measurements in silica/surfactant solutions at very high $\mathrm{pH}$, where extensive condensation of silica is prevented. Their experiments showed that under such conditions addition of silica to a spherical micelle solution promotes phase separation into a dilute phase composed of mainly silica and surfactant monomers and a concentrated phase made up of a silica-surfactant HLC mesostructure. They postulated that HLC formation through this phase separation mechanism is brought about by intermicellar attractions, which in turn arise because of silica adsorption at micellar surfaces. In this so called "strong screening limit", adsorption of anionic silica oligomers neutralizes the electrostatic repulsion between positively charged micelle surfaces and the system becomes dominated by dispersion attractions between micelles. This is in contrast to HLC formation in pure CTAB solutions at high surfactant concentration, which does not involve phase separation and is dominated by electrostatic repulsion between micelles, the so called "weak screening limit". The observation that HLC phases are formed in the presence of small silica oligomers (mainly the double four ring, or D4R species), but not when only monomers are present, led Firouzi et al. to conclude that the charge multiplicity of anionic silicates was crucial for the micelle aggregation process. ${ }^{11}$ Nevertheless, only indirect evidence was presented to support these hypotheses as they were not able to follow detailed, molecular pathways to HLC formation. A further conclusion of their study was that the structure of the mesophase can be changed by adding a cosolvent (in their case either benzene or trimethylbenzene). When cosolvent was added to a previously formed hexagonal phase, this quickly adopted a lamellar morphology. This kind of structural change was only possible under experimental conditions in which extensive silica network formation was inhibited. The molecular statistical mechanics of these important experimental findings have yet to be explained in detail.

In this paper, we present a realistic model that for the first time is able to reproduce all steps leading to the formation of a HLC phase in dilute silica-surfactant solutions in close agreement with the experimental results of Firouzi et al. ${ }^{11}$ Our results shed further light on the molecular level mechanisms at play during mesoporous silica synthesis and open the door for computational predictions of porous material structure from knowledge of the synthesis conditions alone. The paper is organized as follows: section 2 describes the coarse grained (CG) model and the new parameters for silica oligomers and reports simulation details, and section 3 presents the simulation results obtained with our new model in comparison with experiment. Finally, section 4 summarizes the conclusions of our study.

\section{SIMULATION DETAILS AND MODEL DESCRIPTION}

The main aim of our model is to strike the right balance between realism, enabling a direct link with experimental systems and conditions, and computational efficiency, allowing us to reach the time and length scales that are relevant for MCM 41 mesophase structure formation. We aim in particular to reproduce the experimental conditions of Firouzi et al. ${ }^{11}$ As such, we model precursor solutions composed of water, $\mathrm{CTA}^{+}$surfactants, bromide counterions, tetramethylammonium $\left(\mathrm{TMA}^{+}\right)$cations, and silicates with different concentrations. The effect of $\mathrm{pH}$ is implicitly accounted for by considering silicates with different degrees of condensation and ionization according to the experimental compositions determined by NMR experiments. ${ }^{1}$ All simulations take place at room temperature $(300 \mathrm{~K})$ and pressure $(1 \mathrm{bar})$ and were carried out with the Gromacs 4.5.5 package, ${ }^{37-40}$ adopting the leapfrog algorithm ${ }^{41}$ to integrate the equations of motion. Gromacs input files and final configurations for each simulation run are available for download from the University of Strathclyde (DOI: 10.15129/a079acae 9db4 4a62 bb30 11959 fe19f6d). The time step was $30 \mathrm{fs}$ in coarse grained (CG) and $2 \mathrm{fs}$ in all atom (AA) simulations (necessary to calibrate the CG model). The temperature was fixed through the velocity rescaling thermostat, ${ }^{42}$ while the Berendsen pressure coupling method ${ }^{43}$ and the Parrinello-Rahman barostat ${ }^{44}$ were used for CG and AA simulations, respectively. Thermostat and barostat parameters (e.g., frequencies) were set to the same values as in our previous work. ${ }^{34}$ Cubic boxes, periodic boundary conditions, and isotropic pressure scaling were used except where explicitly noted. Unless otherwise stated, initial configurations were prepared by randomly placing all molecules in the simulation box using the PACKMOL software. ${ }^{43}$ In all simulations the same protocol was used: energy minimization using the steepest descent algorithm to prevent short range contacts between atoms; a short (150 ps) equilibration in the NVT ensemble; further equilibration and production run in the $\mathrm{NpT}$ ensemble. The duration of the $\mathrm{NpT}$ stage ranged from a few up to tens of microseconds. We note that for CG simulations a correction factor of approximately four should be applied to yield a realistic time scale ${ }^{36}$ because of the mapping of four particles to one (see below), but this was not applied here, i.e., the time reported in our results is simply the number of steps multiplied by the nominal time step. To analyze the CG simulation results we identified surfactant aggregates using an adaptation of the Hoshen-Kopelman cluster counting algorithm, ${ }^{46}$ considering that two surfactant molecules belong to the same cluster when their tail sites are separated by no more than $0.76 \mathrm{~nm}$, i.e., the first minimum of the respective radial distribution functions.

Bonded interactions - bond length stretching, angle bending, and torsion-together with nonbonded Lennard-Jones (LJ) and Coulombic terms were taken into account in the Hamiltonian of the system. For LJ interactions in both AA and CG representations, a cutoff distance of $1.2 \mathrm{~nm}$ together with a switching function from 0.9 to $1.2 \mathrm{~nm}$ with the standard Gromacs functional form was used. Electrostatic interactions were fully accounted for in AA simulations by the particle mesh Ewald method, ${ }^{47}$ while in the CG simulations a Gromacs standard switching function was employed from 0 to $1.2 \mathrm{~nm}$, as described in the original MARTINI publications. ${ }^{35,36}$ The MARTINI 2.2 force field was adopted in our CG model, taking the default dielectric constant value of $15 .^{36}$ In this paper we take the original MARTINI nomenclature for each CG bead type ( $\mathrm{P}=$ polar; $\mathrm{Q}$ = charged; $\mathrm{C}=$ apolar; $\mathrm{N}=$ nonpolar). This force field adopts an approximate mapping of four heavy atoms per CG bead. As such, each CG water bead represents four atomistic water molecules and is modeled by a standard MARTINI $\mathrm{P}_{4}$ bead. An undesirable artifact of the MARTINI model, arising from the coarse graining of water molecules into Lennard-Jones sites, is that the melting point of water is raised. To avoid unrealistic freezing of the CG model of water, $10 \%$ of water particles were replaced by $\mathrm{BP}_{4}$ antifreeze particles as 
prescribed in the original publications. ${ }^{35,36}$ Antifreeze particles have the same interactions as $\mathrm{P}_{4}$ with all other particles in the system but have a higher excluded volume when interacting with other $\mathrm{P}_{4}$ particles, thus effectively introducing an entropic penalty for freezing. An alternative approach to avoid this issue would be to employ a polarizable coarse grained water model, as proposed by Yesylevskyy et al., ${ }^{48}$ but this would prohibitively increase the computational cost of our already quite demanding simulations. The amphiphilic character of the $\mathrm{CTA}^{+}$surfactant is described by four apolar $\mathrm{C}_{1}$ beads for the tail and one charged (hydrophilic) $\mathrm{Q}_{0}$ bead for cetyltrimethylammonium head groups. ${ }^{49}$ Bromide counterions were represented by charged hydrogen bond acceptor $\mathrm{Q}_{a}$ beads, ${ }^{36}$ while tetramethylammonium $\left(\mathrm{TMA}^{+}\right.$) cations were modeled by single $\mathrm{Q}_{0}$ beads (details of the AA to CG mapping scheme can be consulted in Figure $S 1$ in the Supporting Information). Silica monomers were parametrized against atomistic simulations in our previous work ${ }^{34}$ and are thus represented by a single $\mathrm{Q}_{\mathrm{SI}}$ bead. All parameters for the atomistic simulations were reported in previous publications, ${ }^{15,30,50}$ and the reader is referred to those articles for additional details.

In this paper, we extend our previous CG model ${ }^{34}$ to take into account silicates of a higher degree of condensation (up to octamers ${ }^{11}$ ). To obtain the CG parameters for silica oligomers we used a procedure similar to that in our previous work, ${ }^{34}$ which is based on comparing radial density profiles of preformed micelles in both CG and AA representations. Thus, a spherical $\mathrm{CTA}^{+}$surfactant micelle of aggregation number 100 (corresponding to experimental values ${ }^{51-57}$ ) was first built using PACKMOL, followed by random addition of counterions (in this case, different anionic silicate oligomers) to neutralize the charge, solvation with water, and equilibration. The simulation box size was adjusted such that the average concentration of each system was well above the critical micelle concentration of CTAB $(\mathrm{CMC}=0.8 \mathrm{mM}) .^{58-60}$ Production runs for preformed micelle simulations were $20 \mathrm{~ns}$ long. The equilibrium radial density profiles, measured from the center of mass of the micelle, were then compared in the AA and CG representations, with CG interaction parameters tuned until the AA and CG results fell into reasonable agreement.

As a first guess for the silica dimer parameters we used the same parameters as for monomers, i.e., each dimer was composed of two $Q_{S I}$ beads. However, these parameters were unable to reproduce the interfacial structure observed in the AA simulations, as shown in Figure S4. In fact, the peak for silicates (red lines) is present within the headgroup layer for the CG model, while it is outside the headgroup layer for the AA model. This suggests that the interactions between silicate dimers and surfactants are too attractive. After testing a wide range of parameters we modeled each dimer as being composed of two standard MARTINI $\mathrm{Q}_{\mathrm{da}}$ beads. Using these parameters, excellent agreement with the AA micelle density profiles was achieved, as shown in Figure 1. The same approach was then followed for larger oligomers, with $\mathrm{Q}_{\mathrm{da}}$ beads closely reproducing the AA behavior of linear trimers and tetramers (see Figures S5 and S6). In the case of cyclic oligomers, the $S Q_{d a}$ parameters were used in order to follow the recommendation of Marrink et al. ${ }^{36}$ for ring molecules (detailed AA to CG mapping for silicates is given in Figures S2 and S3). Prefixed "S" beads in MARTINI only change their self interaction; in particular, the sigma value of the Lennard-Jones potential is reduced to $0.43 \mathrm{~nm}$, and epsilon is scaled to $75 \%$ of the original value. In this manner, cyclic molecules can pack more closely to one another without freezing, hence reproducing their corresponding experimental densities. The interactions between prefixed " $S$ " beads and other beads remain unaffected. Once again, good agreement with AA density profiles was obtained for cyclic trimers and tetramers, as well as for the cyclic branched tetramer, which has three $S Q_{d a}$ beads for the ring and one $\mathrm{Q}_{\mathrm{da}}$ bead for the branched segment (Figure S6). Finally, the CG SQ $\mathrm{Q}_{\mathrm{da}}$ beads were also used for all D4R species (Figure S6). Complete tables with the interaction potential parameters for all species studied in this work are provided in the Supporting Information (Tables S1 and S2). In addition, Table $S 3$ provides details of all simulation runs carried out in this work.

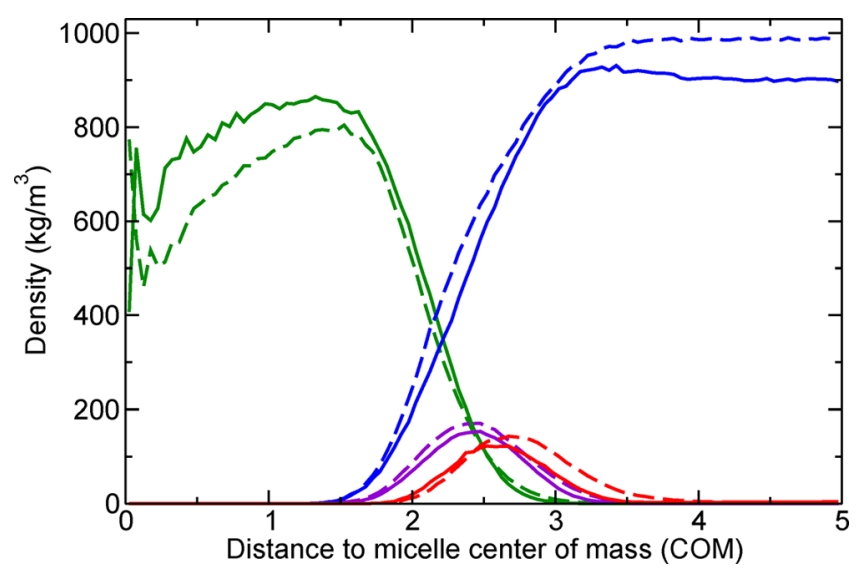

Figure 1. Comparison of radial density profiles for preformed spherical micelles containing silica dimers in the coarse grained (solid lines) and atomistic (dashed lines) representations. Color code is as follows: surfactant tails are shown in green, surfactant heads in purple, silicates in red, and water molecules in blue.

\section{RESULTS AND DISCUSSION}

3.1. Formation of Hexagonal Liquid Crystals. In our previous atomistic simulations we have shown that anionic silica monomers adsorb strongly on the surface of spherical cationic surfactant micelles, replacing bromide counterions in the process. $^{30}$ This strong silica adsorption led to a strong charge density matching with the surfactant head groups, promoting a decrease in curvature and ensuing micelle growth. By coarse graining this atomistic model we showed that the effect of silica monomers was to eventually promote a sphere to rod transition in the system. ${ }^{34}$ The first step of the present work was to coarse grain the atomistic model for anionic silica dimers $^{15}$ in order to examine the effect of more highly condensed silicates on the surfactant structures in solution, considering that Firouzi et al. ${ }^{11}$ hypothesized that silica oligomers are an essential ingredient for HLC formation. To test this hypothesis we compare three simulations at a low surfactant concentration used in the study by Firouzi et al. ${ }^{11}$ ( $6 \%$ by mass, corresponding to the spherical micelle region of the pure CTAB phase diagram ${ }^{61}$ ), starting from random initial configurations in cubic boxes of $30 \mathrm{~nm}$ size. The first simulation (run 1 ) is a reference solution of CTAB in water (4000 ion pairs), the second simulation (run 2) contained $\mathrm{CTA}^{+}$and the same number of silica monomers (replicating the results of our previous study ${ }^{34}$ ), while the third solution (run 3) contains $4000 \mathrm{CTA}^{+}$and 2000 silica dimers. The last two solutions contain a silica/surfactant ratio of 1 . As in previous work, we considered one negative charge per silicon atom, such that each monomer has a charge of -1 and each dimer has a charge of -2 . This amounts to assuming that the solutions are at very high $\mathrm{pH}(\sim 14)$ and neglecting the effect of more highly charged species (for example, doubly charged monomers). The former assumption will be relaxed for work described later in this paper and is used at this point mainly for computational convenience. As for more highly charged species, their concentrations are relatively low at experimentally relevant conditions, $^{62}$ and their neglect is not likely to affect the conclusions of our study.

In Figure 2a, we can see that at equilibrium the reference solution (i.e., without silica) is composed of an isotropic dispersion of small spherical micelles. The average aggregation number of our model surfactant $(\sim 95)$ is in excellent 
a)

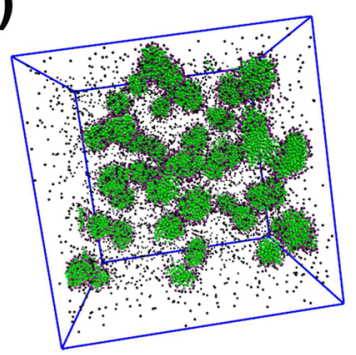

c)

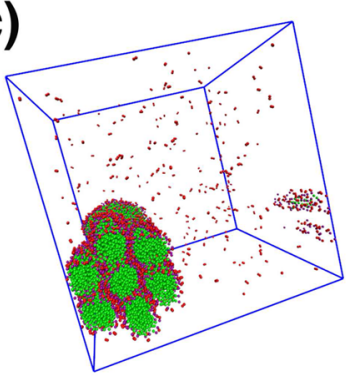

b)

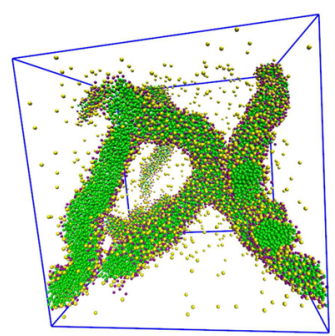

d)

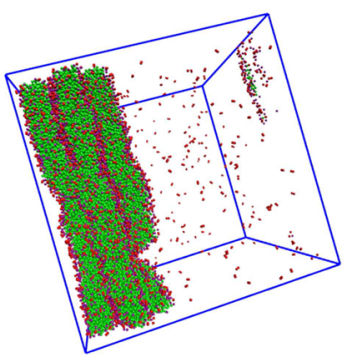

Figure 2. Snapshots of equilibrated simulations for (a) aqueous $\mathrm{CTAB}$ solution, (b) aqueous solution of $\mathrm{CTA}^{+}$and silica monomers, (c) aqueous solution of $\mathrm{CTA}^{+}$and silica dimers (front view), and (d) aqueous solution of $\mathrm{CTA}^{+}$and silica dimers (side view in cross section through the middle of the hexagonal array). Surfactant concentration is $6 \%$ by mass in all solutions, with all molecules randomly distributed at the start and the temperature fixed at $300 \mathrm{~K}$. Color code is as follows: surfactant tails are shown in green, surfactant heads in purple, bromides in black, silica monomers in yellow, and silica dimers in red. Water molecules were removed for a clear visualization.

agreement with experimental data $(90-110) \cdot{ }^{51-57}$ In accord ance with our previous work, ${ }^{34}$ the solution containing silica monomers (shown in Figure $2 \mathrm{~b}$ ) started by forming spherical micelles, but these quickly fused to yield a small number of rod shaped aggregates with some degree of branching (akin to wormlike micelles observed experimentally ${ }^{63-66}$ ). It should be noticed that the wormlike micelle radius shown in Figure $2 \mathrm{~b}$ is $\sim 2.4 \mathrm{~nm}$, which is very close to similar CTAB wormlike micelles $(2.2 \mathrm{~nm})^{66}$ obtained experimentally. Importantly, this system showed no signs of evolving toward a more ordered state, even after $20 \mu$ s of simulation time. This strongly suggests that the simple presence of anionic silica monomers is insufficient to induce the formation of an HLC phase, in agreement with the interpretation of Firouzi et al. ${ }^{11} \mathrm{We}$ will return to this point below.

The more interesting results were found in the silica dimer solution, Figure $2 \mathrm{c}$. In this case, the equilibrium structure was an aggregate of about 7 small rod shaped micelles dispersed in a dilute surfactant monomer solution. These rods were arranged in a honeycomb structure, very reminiscent of the HLC seen experimentally. ${ }^{11}$ Although our simulation conditions do not form materials with experimentally relevant length scales, the fact that a single aggregate was formed in equilibrium with a dilute solution strongly points toward a phase separated system. Importantly, in contrast to the reference (Figure 2a) and monomeric (Figure $2 \mathrm{~b}$ ) solutions, the random dimer solution was very slow to equilibrate, taking almost $20 \mu$ s to converge to the hexagonal micellar array. In the initial stages of this process small surfactant aggregates were quickly formed and fused into micelles after $0.153 \mu \mathrm{s}$ of simulation time. These micelles then grew until they reached an average size close to the experimental value of 100 surfactants per micelle for pure $\mathrm{CTAB}$ systems. After about $2 \mu \mathrm{s}$ of simulation the micelles began to deform, adopting first a prolate shape and later a more elongated rodlike shape. At the same time these deformed micelles started to arrange themselves into several small aggregates of parallel rods, which fused together after $9 \mu \mathrm{s}$ to form a unique aggregate, thus achieving the phase separation. In the final slow step the micellar aggregate became progressively more ordered, reaching the final hexagonal conformation after $20 \mu \mathrm{s}$. A series of snapshots of this process as well as a movie of the entire simulation are provided in the Supporting Information (Film1 and Figure S7).

Although the previous simulations show that silica dimers are able to induce the aggregation of cylindrical micelles to form a hexagonal array it is very important to establish if this is truly an equilibrium configuration and not simply a kinetically arrested state. To verify this we started from the equilibrated monomer solution shown in Figure $2 \mathrm{~b}$ and replaced all monomers by dimers (run 4). More precisely, we first removed all water molecules and silica monomers from the configuration and then randomly distributed 2000 dimers in the available space and resolvated the system. A short equilibration with all surfactant molecules kept fixed using position restraints allowed the dimers to readsorb onto the surface of the undisturbed micellar rods, such that the starting point for further equilibration was a stable rodlike micelle solution with silicate counterions. Soon after all molecules were allowed to move freely, the large surfactant rods began to break up into smaller fragments. These fragments then began to aggregate but without fusing, i.e., the system evolved toward an aggregate of

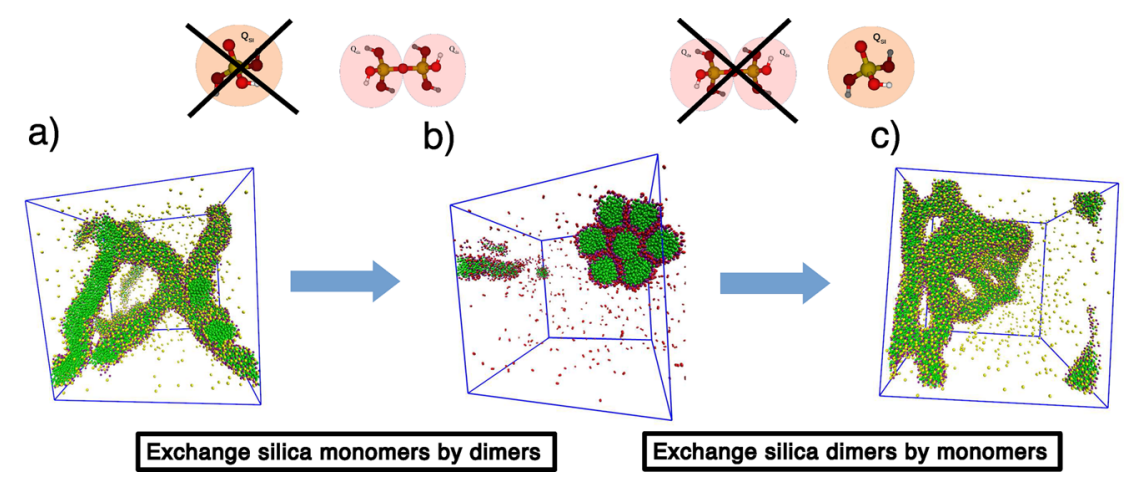

Figure 3. Simulation snapshots obtained in (a) a $\mathrm{CTA}^{+}$and silica monomer solution after equilibration, (b) a solution obtained by replacing all monomers in a by silica dimers, and (c) a solution obtained by replacing all dimers in b by silica monomers. Color code is the same as in Figure 2 . 
micelles. Crucially, the final equilibrium state contained a single hexagonally ordered aggregate of cylindrical micelles, just as in the random solution shown in Figure 2c. This process of replacing monomers by dimers and re equilibrating is depicted in Figure $3 a$ and $3 b$, a process which more realistically represents the real experimental scenario of progressively increasing the degree of silica condensation in the precursor solution (see also Film2 in Supporting Information). Subsequently, we performed the reverse process (run 5) in which we replaced the silicate dimers by monomers (using an analogous procedure as described above and once again ensuring that the system remained overall charge neutral), cf. Figure $3 \mathrm{c}$. The presence of monomers led the small micelles to fuse into very long wormlike micelles, recovering the original equilibrium state. This shows that the HLC phase formed in the dimer solution as well as the rodlike micellar state formed in the monomer solution are true equilibrium configurations and not metastable states. A further interesting observation is that the system depicted in Figure $3 \mathrm{~b}$ equilibrated much faster (in less than $1 \mu \mathrm{s}$ the rods were close to hexagonally arranged) than the one shown in Figure 2c. It appears that the prior existence of a rodlike micellar solution favors subsequent formation of HLC arrays upon silica dimerization. Indeed, during the equilibration of the random dimer solution no intermediate rodlike state was observed (further details can be obtained from Figure S7 and Film1 in the Supporting Information), which is likely to be the cause of the very slow equilibration process for that particular system. It should be emphasized that the more relevant scenario for realistic MCM 41 synthesis solutions is likely to correspond to Figure $3 b$, i.e., the addition of silica monomers promotes micelle growth and fusion into rodlike aggregates, and these subsequently lead to the formation of hexagonal aggregates upon mild silica condensation. In the next section of this paper we study the role of even more highly condensed silica oligomers on the nature of the surfactant mesostructures. Before we do that, however, we examine in more detail the role of silica dimers in promoting micelle aggregation.

In the experimental study of Firouzi et al. ${ }^{11}$ the authors observed the formation of HLC phases from solutions containing silica oligomers but reported the absence of such structures in solutions that contained mostly monomers, more specifically, a solution containing $85 \%$ monomers and $15 \%$ dimers, according to their NMR measurements. This suggests the existence of a minimum concentration of silica in the form of dimers or higher oligomers that is needed to induce HLC formation. To test this hypothesis we carried out simulations (starting from random distributions) of solutions containing mixtures of monomers and dimers in different proportions (runs 6-8). Our model indeed predicts that a solution containing only $15 \%$ dimers (Figure $4 a$ ) does not lead to an HLC phase, in agreement with experiment, ${ }^{11}$ but instead leads to a branched rod configuration. However, when the dimer concentration is increased to $33 \%$, a HLC mesostructure is formed (Figure $4 \mathrm{~b}$ ). Interestingly, this concentration corre sponds to a 50/50 distribution of the negative charge over monomers and dimers (recall that dimers have a charge of -2). As expected, a 50\% concentration of dimers (Figure 4c) also leads to HLC formation. Analyzing this 50\% simulation in more detail we were able to see that the self assembly proceeds in two distinct steps: an initial one involving fast micelle fusion and sphere to rod transitions followed by a slower one involving micelle aggregation, ordering, and phase separation a)

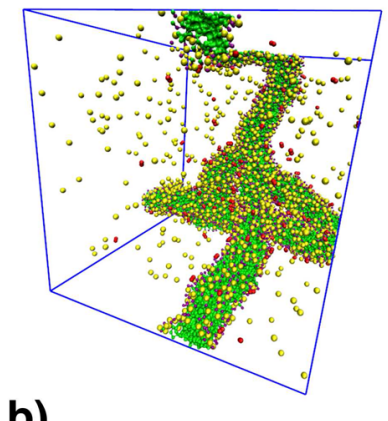

b)
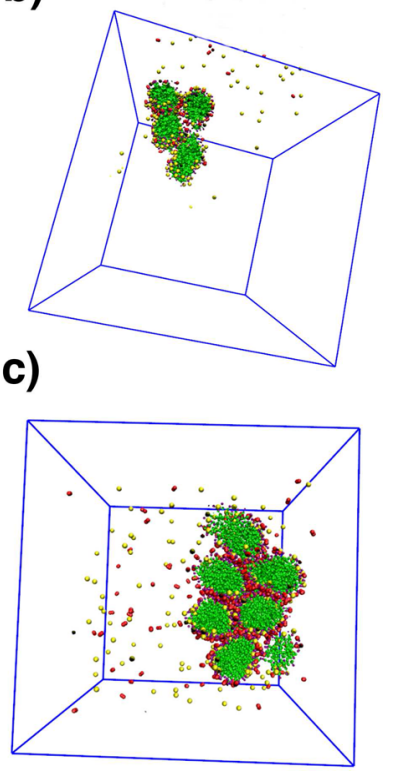

Figure 4. Snapshots obtained from equilibrated simulations of $\mathrm{CTA}^{+}$ solutions with different fractions of silica monomers and dimers: (a) $15 \%$ of dimers and $85 \%$ of monomers, (b) $33 \%$ of dimers and $67 \%$ of monomers, and (c) $50 \%$ of dimers and 50\% of monomers. Selected cross sectional planes are shown in $\mathrm{b}$ and $\mathrm{c}$ for ease of visualization. Color code is the same as in Figure 2.

(Film3 in the Supporting Information shows details of this simulation). This lends further support to our earlier conjecture that HLC formation is favored by the prior existence of large rodlike micelles in the solution, and indeed, the 50\% simulation was much faster to equilibrate $(\sim 2 \mu \mathrm{s})$ than the equivalent simulation containing $100 \%$ dimers, which did not form large rods in the initial stages.

To explain the need for the presence of silica oligomers in order to form HLC phases, Firouzi et al. ${ }^{11}$ conjectured that the oligomers were able to bind to more than one micelle at the same time and thus induce micellar aggregation by effectively screening intermicelle repulsion. Preliminary evidence in support of this hypothesis was obtained in our earlier atomistic simulation studies, ${ }^{15}$ although those studies were limited to small sizes and time scales. With our new CG model we examined the relative propensity of silicate monomers and dimers to bind to more than one micelle simultaneously in an HLC mesostructure. In Figure 5 we show a cross section of the configuration previously shown in Figure $4 c$ but with all surfactant and water molecules removed. The cross sectional plane was chosen to be roughly perpendicular to the vector that goes along the cylindrical micelles in the HLC phase, so as to show the internal structure of that aggregate. When only the 
a)

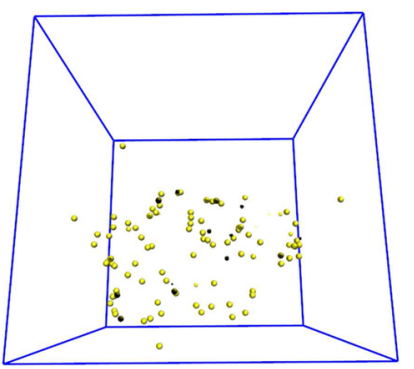

b)

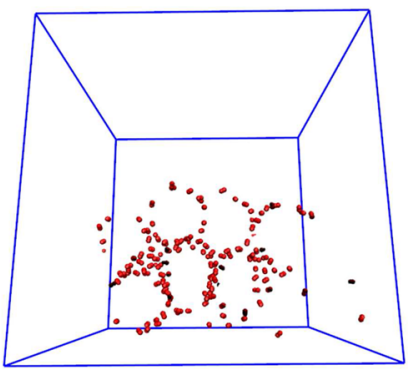

Figure 5. Snapshots of the simulation corresponding to Figure $4 \mathrm{c}$ but showing only (a) silica monomers in yellow which show a roughly homogeneous distribution and (b) silica dimers in red which show beginnings of hexagonal ordering. Water and surfactant molecules were removed for clearer visualization.

silica monomers are shown these are seen to adopt a nearly uniform distribution around each cylindrical micelle belonging to the HLC phase (Figure 5a). In contrast, silica dimers (Figure $5 b)$ are located mainly at the interfaces between adjacent micelles in the HLC structure. In fact, a detailed analysis of thin (one bead thick) slices of the HLC phase allowed us to conclude that only dimers are able to bind to two different rods, whereas monomers are adsorbed within the headgroup region of individual micelles (see Figure S8). This supports the notion of micelle aggregation induced by multidentate binding by silica oligomers.

3.2. Larger Silicate Oligomers. In the previous section we showed that silica monomers and dimers behave differently in the self assembly process of silica/surfactant systems and that multidentate binding by silica dimers to surfactant micelles is required to promote HLC formation. Most realistic precursor solutions to MCM 41 include a distribution of small silica oligomers (up to at least octamers), and in this section we analyze the effect of larger oligomers on the structure of the equilibrium mesophase. To speed up the computations we simulated a somewhat higher surfactant concentration $(20 \%$ by weight), which is nevertheless still within the micellar region of the pure surfactant phase diagram. ${ }^{61}$ We also employed elongated simulation boxes $(8 \times 8 \times 34 \mathrm{~nm})$, instead of cubic boxes as previously, to promote faster phase separation. For the time being we maintain the previous assumption that all oligomers contain a single negative charge per silicon atom. Figure 6 shows the results of $6 \mu$ s simulations starting from fully random configurations for solutions containing $100 \%$ of dimers (run 9; Figure 6a), cyclic trimers (run 10; Figure 6b), cyclic tetramers (run 11; Figure 6c), and cubic octamers (run 12; Figure 6d).

These species are chosen as typical examples of highly condensed small anionic oligomers present in MCM 41 precursor solutions at high $\mathrm{pH} .^{11,17,67}$ The first thing to notice is that the system with dimers produced a two phase arrangement composed of a dilute solution and a hexagonal mesostructure in the surfactant rich phase. This confirms our previous assertion that the presence of dimers induces phase separation and triggers the formation of HLC mesophases. When the silicate oligomers are in the form of cyclic trimers, a similar arrangement of micelles is found. However, it is possible to see that the cross section of the elongated micelles forming the hexagonal arrangement is more elliptical in shape (Figure 6b) than those formed with dimers (Figure 6a). A more a)

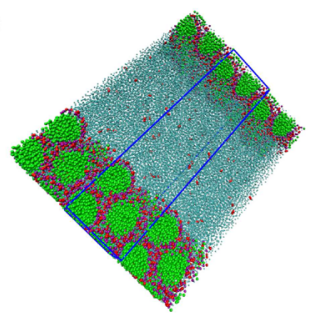

b)

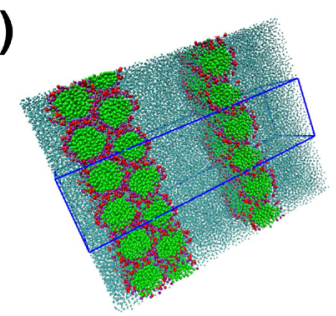

c)

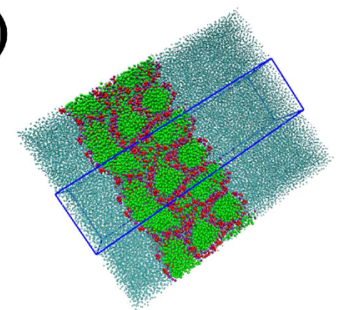

d)

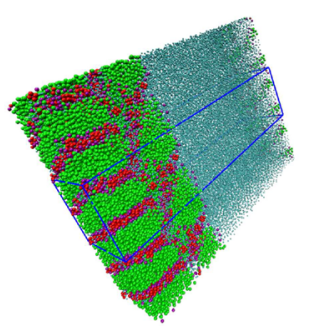

Figure 6. Snapshots of simulations of $20 \% \mathrm{w} / \mathrm{w} \mathrm{CTA}^{+}$solutions with anionic silicates of increasing degree of condensation: (a) dimers, (b) cyclic trimers, (c) cyclic tetramers, and (d) cubic octamers. Color code is the same as in Figure 2 with all silica oligomers shown in red. Notice that elongated boxes were used to facilitate phase separation. Periodic replicas of the central box (blue lines) are shown to enhance the perception of the resulting phases.

significant difference is observed when silicates are in the form of square shaped tetramers. In this case, although the system still forms a phase separated aggregate of elongated micelles, the shape of this aggregate is much more disordered than before. It is possible to see (Figure 6c) that the micelles are still arranged in a more or less hexagonal order, but their cross sections depart significantly from circular. Finally, when all silicates are in the form of fully deprotonated cubic octamers, the mesophase assumes the structure of disordered lamellae (Figure 6d). This gradual transformation is caused by the progressive increase in the charge density of the silicate counterions as we move from dimers to octamers. Because the total charge remains constant to preserve charge neutrality, a single cubic octamer will have the same charge as four dimers but will be concentrated over a much smaller volume. As discussed by Israelachvili, ${ }^{68}$ this increased charge density reduces the effective area per headgroup of the surfactants, thus reducing the curvature of the micelles. The net result is a gradual shift toward less curved shapes (cylinders to lamellae) as the charge density of counterions increases. 
An alternative way to change the charge density is by modifying the degree of deprotonation of silicate oligomers. To test this scenario we carried out three simulations of solutions containing $100 \%$ cubic octamers but each with a different molecular charge: -4 (run 13), -6 (run 14), and -8 (run 12). As before, overall charge neutrality is maintained, so the system composed of $\mathrm{D} 4 \mathrm{R}^{-4}$ contains twice as many silicate species as the one composed of $\mathrm{D} 4 \mathrm{R}^{-8}$. The results are presented in Figure 7 , where we can see that as the charge on individual

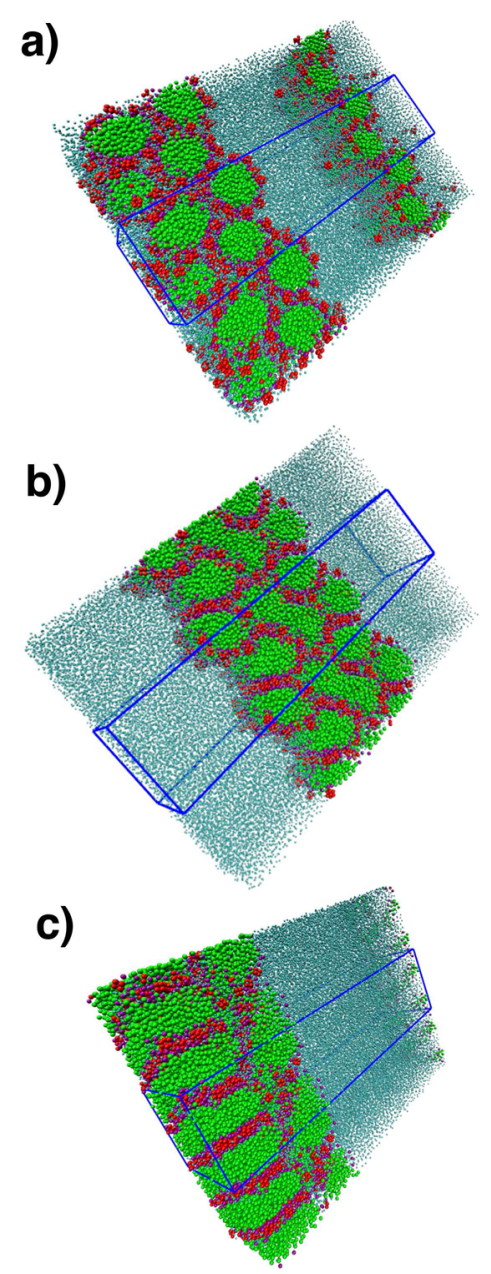

Figure 7. Snapshots of simulations of $20 \% \mathrm{w} / \mathrm{w} \mathrm{CTA}^{+}$solutions with cubic silicate octamers (D4R) with increasing degree of ionization: (a) charge of -4 per molecule, (b) charge of -6 per molecule, and (c) charge of -8 per molecule. Color code and setup are the same as in Figure 6.

octamers decreases the system gradually shifts from a lamellar phase (Figure 7c) to a hexagonal arrangement of cylindrical micelles (Figure 7a). The physical principle behind this transition is analogous to the one shown in Figure 6; the $\mathrm{D} 4 \mathrm{R}^{-4}$ species have much lower charge density than the fully charged octamers and therefore yield structures with a higher degree of curvature.

Our results obtained in simplified precursor solutions (e.g., containing a single type of silicate) show that both the degree of condensation and the degree of deprotonation of silicate oligomers can have a profound effect on the formation of liquid crystal phases and on the particular structure of those phases. In the final simulation of this section we examine a more realistic system, corresponding to the base case in the study of Firouzi et al. ${ }^{11}$ Our starting point is a pre equilibrated solution of $\mathrm{CTAB}$ in water at $6 \%$ concentration by weight (i.e., in the spherical micelle region of the phase diagram). To this system we randomly added a distribution of silicates corresponding to the experimental ${ }^{29} \mathrm{Si} \mathrm{NMR}$ measurements in the dense surfactant rich phase after addition of TEOS at a $\mathrm{pH}$ of about 13. The experimental data (see Figure 4 of Firouzi et al.) shows that the solution contains $70 \%$ of D4R species, $23 \%$ of monomers, and $7 \%$ of prismatic hexamers in molar percentage. Because the concentration of the last species is rather low we simplified the simulation composition by removing the prismatic hexamers and then renormalizing the concentrations of monomers and octamers. The result is a distribution containing $75 \%$ of octamers and $25 \%$ of monomers. We also considered a higher silica/surfactant ratio of 3 (instead of 1 as in all our previous simulations) to match the experimental conditions. Finally, we relaxed our previous assumption of full deprotonation of each silicon containing group and instead used experimental information on the deprotonation equilibra of monomers ${ }^{62}$ and more highly condensed oligomers. ${ }^{11}$ Estimates based on the above data yield a charge of -1 for each monomer and a charge of (at most) -6 for each octamer. ${ }^{11}$ The excess negative charge of the silicates is compensated by adding the appropriate number of $\mathrm{TMA}^{+}$ions. Figure 8 shows the result of this simulation (run a)

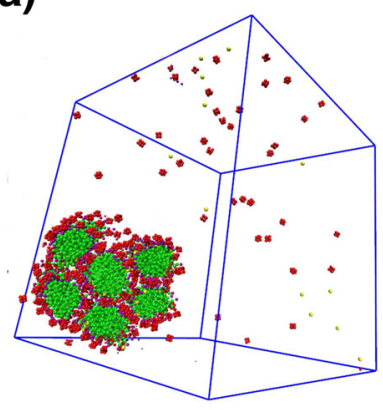

b)

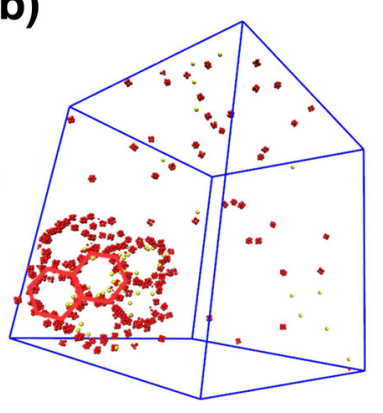

Figure 8. Snapshots of an equilibrated simulation corresponding to a realistic solution studied in the experimental work of Firouzi et al. ${ }^{11}$ The surfactant concentration is $6 \%$ by weight, the silica/surfactant ratio is 3 , silicates are in the form of $75 \% \mathrm{D} 4 \mathrm{R}^{-6}$ and $25 \%$ singly charged monomers, and the temperature is $300 \mathrm{~K}$. (a) Cross section of the resulting mesophase with all water molecules removed. (b) Same cross section but removing also the surfactant molecules to show the hexagonal ordering of the silica layers (highlighted by the red lines). Color code is the same as in Figure 2.

15). After about $1.3 \mu \mathrm{s}$, the system phase separated into a silica/ surfactant mesophase in equilibrium with a dilute solution. The mesophase consisted of an aggregate formed by several twisted rods in a nearly hexagonal arrangement after $9 \mu \mathrm{s}$. The results in Figure 8 indicate that the above simulation is likely the most realistic molecular depiction to date of MCM 41 mesophase formation.

3.3. Effect of Cosolvent Addition. Here we simulate the transition between hexagonal and lamellar mesophases upon addition of a cosolvent, observed experimentally by Firouzi et al. ${ }^{11}$ By adding either benzene or trimethylbenzene to a preformed HLC solution they were able to induce a hexagonal to lamellar transition, which was then reversed by increasing the temperature of the system. They hypothesized that the 
forward transition was due to the incorporation of benzene inside the tail group region, which effectively increased the hydrophobic tail volume and led to lower curvature shapes, ${ }^{68}$ i.e., a lamellar mesostructure. Importantly, they identified a threshold concentration of 0.35 mol \% benzene, below which the transition was not observed. Their hypothesis for the reverse transition was that an increase in temperature caused an increase in the solubility of benzene in water, thus reducing its concentration in the tail region, consequently leading to an increase in curvature.

To test if our model reproduces this behavior we randomly added benzene molecules in their coarse grained representation (see Figure S1c) to the bulk phase of a pre equilibrated silicasurfactant HLC solution with $100 \%$ of silica dimers (Figure 9a). The overall concentration of benzene used in our simulation (run 16) was $2.0 \mathrm{~mol} \%$, which is above the experimentally identified threshold. ${ }^{11}$ As soon as the system started to equilibrate, the benzene molecules migrated to the tail group region of the HLC phase. After $0.5 \mu \mathrm{s}$, the HLC had completely a)

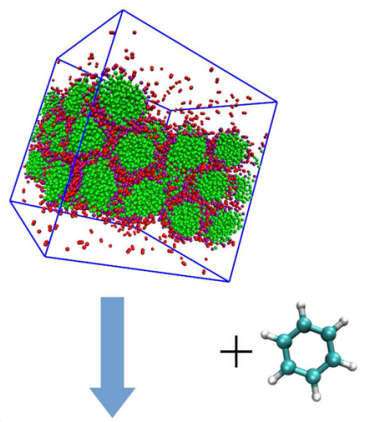

b)

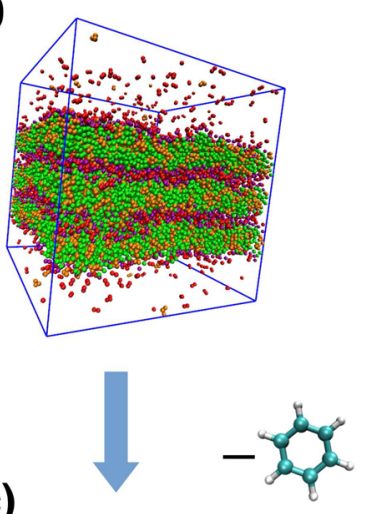

c)

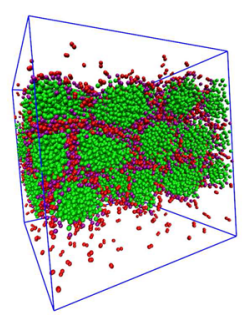

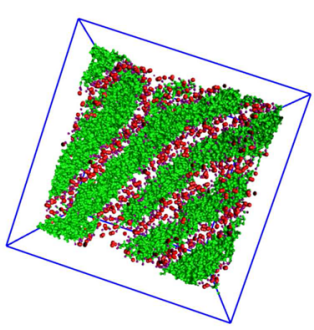
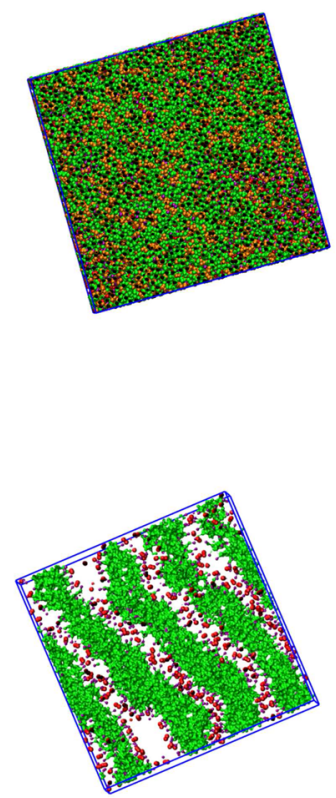

Figure 9. Simulation snapshots obtained in (a) an equilibrated $2.7 \mathrm{~mol}$ $\% \mathrm{CTA}^{+}$solution with $100 \%$ silica dimers, (b) the same solution as a after adding $2.0 \mathrm{~mol} \%$ of benzene and re equilibrating, and (c) the same solution as b after removing all of the benzene molecules and re equilibrating. Figures on the right side show cross sections of each system taken along the plane parallel to the main mesophase axis. Color code is the same as in Figure 2 with CG benzene particles shown in brown. Water molecules are removed for clearer visual ization. transformed into a lamellar mesophase, as can be seen in Figure $9 \mathrm{~b}$. When benzene molecules were deleted from the simulation (run 17), the system reverted back to an HLC (Figure 9c). A movie containing details of the hexagonal to lamellar transition and the reverse process after removing benzenes can be found in the Supporting Information section (Film5 and Film6).

We also calculated the density profile of each type of bead along the most relevant axis of the simulation box for the systems before and after addition of benzene (Figure 10). The

a)

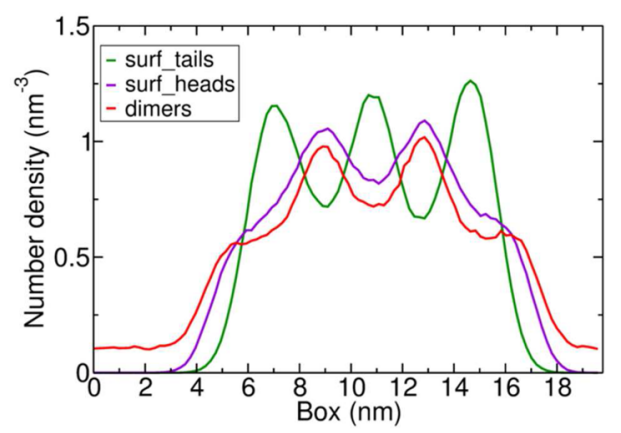

b)

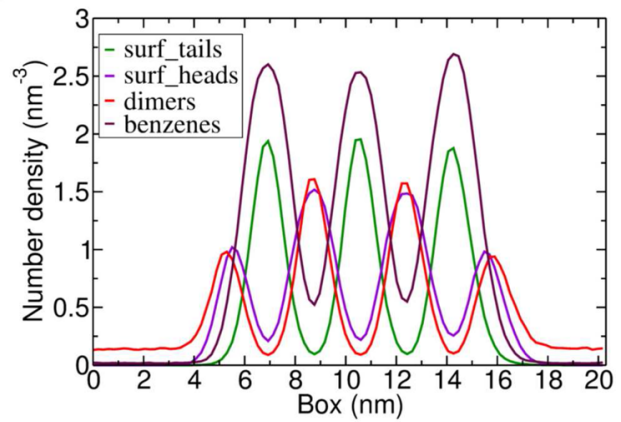

c)

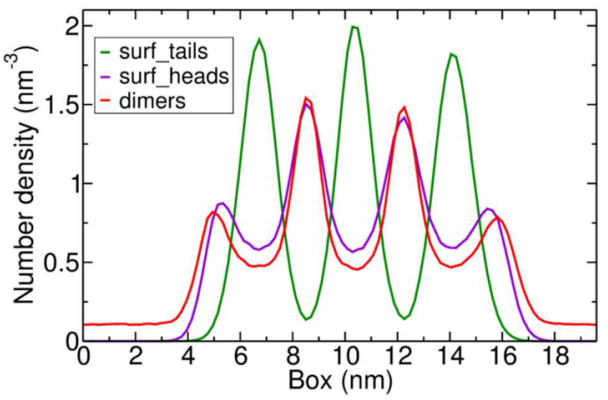

Figure 10. Number density profiles along the $z$ axis of the simulation box for the simulations shown in Figure 9: (a) hexagonal system prior to the addition of benzene, (b) lamellar system after addition of benzene, and (c) hexagonal system after removal of benzene. Green lines represent the terminal beads of the surfactant tail, purple was used for surfactant heads, red was used for silica dimers, and brown was used for benzene.

ordered arrangement of the cylindrical micelles and lamellae appears as evenly spaced peaks in the head and tail group distributions. Examining first the density profiles of the lamellar phase induced by the addition of benzene (Figure 10b) it is possible to confirm that the vast majority of benzene molecules (in brown) are incorporated within the hydrophobic region of the lamellae (see also Figure S9). We estimated the hydrophobic surfactant tail volume contribution before and after adding benzene in the hexagonal and lamellar phases, 
respectively. Details of the calculation procedure are provided in the Supporting Information (Figure S10). Our estimate for the hydrophobic tail volume is $0.586 \mathrm{~nm}^{3} /$ tail for the HLC and $0.891 \mathrm{~nm}^{3} /$ tail for the lamellar system. This means that the presence of benzene molecules brings about an increase of $0.305 \mathrm{~nm}^{3} /$ tail in the effective surfactant tail volume. This is quite a significant increase, particularly in light of the fact that the average molecular volume of benzene in the pure liquid phase for the MARTINI model is only $0.180 \mathrm{~nm}^{3} /$ molecule (based on a liquid density of $0.72 \mathrm{~g} / \mathrm{cm}^{3}$ obtained by Marrink et al. ${ }^{36}$ ). Furthermore, no specific interactions between benzene molecules and surfactant head groups was observed, so it is reasonable to assume that the average area per headgroup is not significantly different in both systems. Taken together, these observations support the experimental hypothesis of Firouzi et al. ${ }^{11}$ that the incorporation of benzene molecules promotes a decrease in curvature of the mesophase by expanding the effective volume of the tail region.

Examining now the profiles for the HLC structures (Figure $10 \mathrm{a}$ and 10c), an important observation is that the silica dimer peaks overlap with the headgroup peaks, further confirming that silica oligomers act as bridges between adjacent micelles. In this regard, the profiles show some artificial broadening (much more prominent in Figure 10a) due to the fact that the axis of the cylindrical micelles in the HLC does not always correspond with the vertical axis of the simulation box. Nevertheless, using the distance between successive silica peaks it is possible to estimate the pore size of the MCM 41 material that would be formed from this HLC mesophase after silica condensation and calcination. We obtained a value of $37 \AA$, which is in reasonable agreement with experimentally reported MCM 41 pore diameter estimates that lie in the range of $35-45 \AA \AA^{63,69-75}$ It should be noticed that our system is the initial HLC formation stage with only silica dimers whereby further silica oligomeriza tion and the increase of the silica density between rods may slightly vary the pore diameter as experimentally observed. ${ }^{11}$ To compare our estimate with the HLC structures obtained by Firouzi et al. ${ }^{11}$ we use the lattice spacing $d_{100}$ determined from their $\mathrm{X}$ ray diffraction measurements and assume a wall thickness $\Delta=1.1 \mathrm{~nm} .^{76}$ The unit cell constant $a_{0}$ is defined as follows

$$
a_{0}=\frac{2 d_{100}}{\sqrt{3}}
$$

where $d_{100}$ is the Bragg peak measured by $\mathrm{X}$ ray. Therefore, the pore diameter can be obtained through ${ }^{76}$

$$
a_{0}=\Delta+0.95 \times \text { diameter }
$$

Firouzi et al. obtained a value of $d_{100}=47.1 \AA$ for the HLC mesophase, which using the relations in eqs 1 and 2 leads to a pore size of $\sim 45 \AA$, which is in reasonable agreement with our modeling results, considering the nature of the assumptions involved and the fact that our system is in the initial HLC formation stage with only dimers. This near quantitative agreement with experiment is a further indication that our model is accurately representing the real synthesis process.

\section{CONCLUSIONS}

We presented a new mesoscale model for precursor solutions used in the synthesis of MCM 41 mesoporous silica materials. The model is based on a multiscale approach, whereby information from quantum chemical calculations ${ }^{32}$ was used to develop a classical atomistic model, ${ }^{15}$ which in turn was used to calibrate the interaction potentials at the coarse grained level. This allowed us to reach the relevant time and length scales for the formation of silica/surfactant mesophases while maintaining sufficient levels of realism to allow close comparisons with experimental studies. Indeed, the results of our simulations are in remarkable agreement with experimental observations of Chmelka and co workers in their detailed studies of MCM 41 formation at high $\mathrm{pH} .{ }^{11,77}$ To our knowledge it is the first time that a molecular model has been able to reproduce the formation of hexagonal liquid crystal structures in realistic aqueous solutions of silica and cationic surfactants. As such our work bridges the gap between previous atomistic modeling efforts that were limited by large computational cost and lattice based simulations that were limited in their relationship to realistic solutions and structures. Although the force field we employed is specific to MCM 41, the multiscale methodology is generally applicable to the synthesis of other templated porous materials with appropriate adaptations. It therefore constitutes a valuable tool for computational material design.

Our simulation results showed that the addition of silica monomers to a low concentration surfactant solution induces the fusion of small spherical micelles to form elongated rodlike aggregates. However, the presence of monomers alone is not sufficient to promote the formation of an HLC phase. Instead, the formation of this phase at low concentration was only observed when a minimum percentage of silicates (between $15 \%$ and $33 \%$ ) was present in the form of dimers or higher oligomers. Such highly condensed anionic silica oligomers have been shown to be stable in precursor solutions, both experimentally $^{78}$ and theoretically. ${ }^{79}$ When anionic silicates are in oligomeric form they are able to act as multidentate binding sites, essentially bridging the headgroup regions of different micelles. These silica bridges counteract the electro static repulsion between micelles, promoting micelle aggrega tion. Crucially, this leads to separation of the system into a dilute solution and a concentrated silica/surfactant ordered mesophase, as observed experimentally. Our model also predicts that the structure of this mesophase can be controlled by varying the charge density of the silicate oligomers, by increasing either their degree of condensation or their degree of deprotonation, with higher silica charge density leading to structures with a lower degree of curvature (e.g., lamellae). This result implies that the mesostructure is rather labile at this stage of the synthesis, before extensive silica condensation takes place, in agreement with previous lattice model predictions. ${ }^{16}$ The degree of flexibility of the mesophase can be exploited by changing the composition of the synthesis solution, and our model was also able to describe the reversible hexagonal to lamellar transition observed experimentally upon addition of a cosolvent. ${ }^{11}$ In this case incorporation of aromatic molecules inside the hydrophobic region of the HLC causes an increase of the effective volume of the surfactant tails, leading to a decrease in the curvature of the aggregate.

The results of our work provide further support for a cooperative templating mechanism in the synthesis of periodic mesoporous silica, whereby the presence of silica drives the formation of a phase separated ordered mesostructure. We demonstrated that the structure of the mesophase is strongly dependent on the composition of the synthesis solution, particularly on the degree of condensation and deprotonation of silicate species. However, we still relied on experimental data (in this case, detailed NMR measurements) to predefine the 
condensation and deprotonation state of silicates in our simulations, and thus, our model is not yet able to predict in an "ab initio" sense the complete formation of mesoporous silica structures. We are currently combining our coarse grained model with the reactive Monte Carlo formalism proposed by Malani et al. ${ }^{80,81}$ to explicitly describe the condensation of silica. We expect that this will lead to further insights into the formation mechanism of this fascinating class of porous materials.

\section{ASSOCIATED CONTENT}

\section{Supporting Information}

The Supporting Information is available free of charge on the ACS Publications website at DOI: 10.1021/acs.chemma ter.6b00348.

Details on the development and validation of the multiscale model for MCM 41 precursor synthesis solutions; full list of potential parameters and simulation details; additional figures and analysis of simulation data (PDF)

\section{AUTHOR INFORMATION}

\section{Corresponding Author}

*E mail: miguel.jorge@strath.ac.uk. Phone: +44 (0)141 548 2825.

\section{Present Address}

"S. C.C.: Department of Materials Science and Engineering, Massachusetts Institute of Technology, Cambridge, Massachu setts 02139, United States.

\section{Notes}

The authors declare no competing financial interest.

\section{ACKNOWLEDGMENTS}

G. P. S., M. J., and J. R. B. G. are thankful for financial support of the PTDC/QUI QUI/109914/2009 project. This work was developed within the scope of the projects CICECO Aveiro Institute of Materials, POCI 010145 FEDER 007679 (FCT Ref. UID/CTM/50011/2013), LAQV@REQUIMTE (UID/ QUI/50006/2013), and LSRE (UID/EQU/500230/2013), financed by national funds through the FCT/MEC and when appropriate cofinanced by FEDER under the PT2020 Partner ship Agreement. G.P.S. acknowledges CICECO UID/CTM/ 50011/2013 Ref. BI/UI89/7145/2015. M.J. acknowledges funding from the EPSRC UK Project Grant EP/L014297/1. This work was produced with the support of the Portuguese National Distributed Computing Infrastructure (INGRID). More information can be found at http://www.incd.pt. P.A.M., S.M.A., and S. C.C. acknowledge generous funding from the US Department of Energy under contract number DEFG02 07ER46466 and the computational resources provided from the Massachusetts Green High Performance Computing Center (MGHPCC).

\section{REFERENCES}

(1) Barton, T. J.; Bull, L. M.; Klemperer, W. G.; Loy, D. A.; McEnaney, B.; Misono, M.; Monson, P. A.; Pez, G.; Scherer, G. W.; Vartuli, J. C.; Yaghi, O. M. Tailored Porous Materials. Chem. Mater. 1999, 11, 2633-2656.
(2) Auerbach, S. M.; Ford, M. H.; Monson, P. New insights into zeolite formation from molecular modeling. Curr. Opin. Colloid Interface Sci. 2005, 10, 220-225.

(3) Van Speybroeck, V.; Hemelsoet, K.; Joos, L.; Waroquier, M.; Bell, R. G.; Catlow, C. R. A. Advances in theory and their application within the field of zeolite chemistry. Chem. Soc. Rev. 2015, 44, 7044-7111.

(4) Raman, N. K.; Anderson, M. T.; Brinker, C. J. Template Based Approaches to the Preparation of Amorphous, Nanoporous Silicas. Chem. Mater. 1996, 8, 1682-1701.

(5) Scott, B. J.; Wirnsberger, G.; Stucky, G. D. Mesoporous and Mesostructured Materials for Optical Applications. Chem. Mater. 2001, $13,3140-3150$

(6) Tanev, P. T.; Chibwe, M.; Pinnavaia, T. J. Titanium containing mesoporous molecular sieves for catalytic oxidation of aromatic compounds. Nature 1994, 368, 321.

(7) Botella, P.; Corma, A.; Quesada, M. Synthesis of ordered mesoporous silica templated with biocompatible surfactants and applications in controlled release of drugs. J. Mater. Chem. 2012, 22, 6394-6401.

(8) Kresge, C. T.; Roth, W. J. The discovery of mesoporous molecular sieves from the twenty year perspective. Chem. Soc. Rev. 2013, 42, 3663-3670.

(9) Beck, J. S.; Vartuli, J. C.; Roth, W. J.; Leonowicz, M. E.; Kresge, C. T.; Schmitt, K. D.; Chu, C. T. W.; Olson, D. H.; Sheppard, E. W. A new family of mesoporous molecular sieves prepared with liquid crystal templates. J. Am. Chem. Soc. 1992, 114, 10834-10843.

(10) Kresge, C. T.; Leonowicz, M. E.; Roth, W. J.; Vartuli, J. C.; Beck, J. S. Ordered mesoporous molecular sieves synthesized by a liquid crystal template mechanism. Nature 1992, 359, 710.

(11) Firouzi, A.; Atef, F.; Oertli, A. G.; Stucky, G. D.; Chmelka, B. F. Alkaline Lyotropic Silicate Surfactant Liquid Crystals. J. Am. Chem. Soc. 1997, 119, 3596-3610.

(12) Ying, J. Y.; Mehnert, C. P.; Wong, M. S. Synthesis and Applications of Supramolecular Templated Mesoporous Materials. Angew. Chem., Int. Ed. 1999, 38, 56-77.

(13) Blin, J. L.; Imperor Clerc, M. Mechanism of self assembly in the synthesis of silica mesoporous materials: in situ studies by $\mathrm{X}$ ray and neutron scattering. Chem. Soc. Rev. 2013, 42, 4071-4082.

(14) Li, W.; Zhao, D. An overview of the synthesis of ordered mesoporous materials. Chem. Commun. 2013, 49, 943-946.

(15) Jorge, M.; Gomes, J. R. B.; Cordeiro, M. N. D. S.; Seaton, N. A. Molecular Dynamics Simulation of the Early Stages of the Synthesis of Periodic Mesoporous Silica. J. Phys. Chem. B 2009, 113, 708-718.

(16) Jin, L.; Auerbach, S. M.; Monson, P. A. Simulating the Formation of Surfactant Templated Mesoporous Silica Materials: A Model with Both Surfactant Self Assembly and Silica Polymerization. Langmuir 2013, 29, 766-780.

(17) Monnier, A.; Schuth, F.; Huo, Q. S.; Kumar, D.; Margolese, D.; Maxwell, R. S.; Stucky, G. D.; Krishnamurty, M.; Petroff, P.; Firouzi, A.; Janicke, M.; Chmelka, B. F. Cooperative Formation of Inorganic Organic Interfaces in the Synthesis of Silicate Mesostructures. Science 1993, 261, 1299-1303.

(18) Frasch, J.; Lebeau, B.; Soulard, M.; Patarin, J.; Zana, R. In Situ Investigations on Cetyltrimethylammonium Surfactant/Silicate Sys tems, Precursors of Organized Mesoporous MCM 41 Type Siliceous Materials. Langmuir 2000, 16, 9049-9057.

(19) Zana, R.; Frasch, J.; Soulard, M.; Lebeau, B.; Patarin, J. Fluorescence Probing Investigations of the Mechanism of Formation of Organized Mesoporous Silica. Langmuir 1999, 15, 2603-2606.

(20) Regev, O. Nucleation Events during the Synthesis of Mesoporous Materials Using Liquid Crystalline Templating. Langmuir 1996, 12, 4940-4944.

(21) Lee, Y. S.; Surjadi, D.; Rathman, J. F. Effects of Aluminate and Silicate on the Structure of Quaternary Ammonium Surfactant Aggregates. Langmuir 1996, 12, 6202-6210.

(22) Albuquerque, A.; Vautier Giongo, C.; Pastore, H. Physical chemistry of nanostructured molecular sieves by the study of phase diagrams: the case of the cetyltrimethylammonium bromide 
tetramethylammonium silicate water system. J. Colloid Interface Sci. 2005, 284, 687-693.

(23) Galarneau, A.; Renzo, F. D.; Fajula, F.; Mollo, L.; Fubini, B.; Ottaviani, M. Kinetics of Formation of Micelle Templated Silica Mesophases Monitored by Electron Paramagnetic Resonance. J. Colloid Interface Sci. 1998, 201, 105-117.

(24) Sadasivan, S.; Fowler, C. E.; Khushalani, D.; Mann, S. Nucleation of MCM 41 Nanoparticles by Internal Reorganization of Disordered and Nematic Like Silica Surfactant Clusters. Angew. Chem., Int. Ed. 2002, 41, 2151-2153.

(25) Auerbach, S. M.; Fan, W.; Monson, P. A. Modeling the assembly of nanoporous silica materials. Int. Rev. Phys. Chem. 2015, 34, 35-70.

(26) Siperstein, F. R.; Gubbins, K. E. Synthesis and Characterization of Templated Mesoporous Materials Using Molecular Simulation. Mol. Simul. 2001, 27, 339-352.

(27) Siperstein, F. R.; Gubbins, K. E. Phase Separation and Liquid Crystal Self Assembly in Surfactant Inorganic Solvent Systems. Langmuir 2003, 19, 2049-2057.

(28) Larson, R. G.; Scriven, L. E.; Davis, H. T. Monte Carlo simulation of model amphiphile oil water systems. J. Chem. Phys. 1985, 83, 2411-2420.

(29) Vartuli, J. C.; Schmitt, K. D.; Kresge, C. T.; Roth, W. J.; Leonowicz, M. E.; McCullen, S. B.; Hellring, S. D.; Beck, J. S.; S, J. L. Effect of Surfactant/Silica Molar Ratios on the Formation of Mesoporous Molecular Sieves: Inorganic Mimicry of Surfactant Liquid Crystal Phases and Mechanistic Implications. Chem. Mater. 1994, 6, 2317-2326.

(30) Jorge, M.; Gomes, J. R. B.; Cordeiro, M. N. D. S.; Seaton, N. A. Molecular Simulation of Silica/Surfactant Self Assembly in the Synthesis of Periodic Mesoporous Silicas. J. Am. Chem. Soc. 2007, 129, 15414-15415.

(31) Pereira, J. C. G.; Catlow, C. R. A.; Price, G. D. Ab Initio Studies of Silica Based Clusters. Part I. Energies and Conformations of Simple Clusters. J. Phys. Chem. A 1999, 103, 3252-3267.

(32) Gomes, J. R.; Cordeiro, M. N. D.; Jorge, M. Gas phase molecular structure and energetics of anionic silicates. Geochim. Cosmochim. Acta 2008, 72, 4421-4439.

(33) Baute, D.; Frydman, V.; Zimmermann, H.; Kababya, S.; Goldfarb, D. Properties of the Silica Layer during the Formation of MCM 41 Studied by EPR of a Silica Bound Spin Probe. J. Phys. Chem. B 2005, 109, 7807-7816.

(34) Pérez Sánchez, G.; Gomes, J. R. B.; Jorge, M. Modeling Self Assembly of Silica/Surfactant Mesostructures in the Templated Synthesis of Nanoporous Solids. Langmuir 2013, 29, 2387-2396.

(35) Marrink, S. J.; de Vries, A. H.; Mark, A. E. Coarse Grained Model for Semiquantitative Lipid Simulations. J. Phys. Chem. B 2004, 108, 750-760.

(36) Marrink, S. J.; Risselada, H. J.; Yefimov, S.; Tieleman, D. P.; de Vries, A. H. The MARTINI Force Field: Coarse Grained Model for Biomolecular Simulations. J. Phys. Chem. B 2007, 111, 7812-7824.

(37) Berendsen, H.; van der Spoel, D.; van Drunen, R. GROMACS: A message passing parallel molecular dynamics implementation. Comput. Phys. Commun. 1995, 91, 43-56.

(38) Lindahl, E.; Hess, B.; van der Spoel, D. GROMACS 3.0: A package for molecular simulation and trajectory analysis. J. Mol. Model. 2001, 7, 306-317.

(39) Van Der Spoel, D.; Lindahl, E.; Hess, B.; Groenhof, G.; Mark, A. E.; Berendsen, H. J. C. GROMACS: Fast, flexible, and free. J. Comput. Chem. 2005, 26, 1701-1718.

(40) Hess, B.; Kutzner, C.; van der Spoel, D.; Lindahl, E. GROMACS 4: Algorithms for Highly Efficient, Load Balanced, and Scalable Molecular Simulation. J. Chem. Theory Comput. 2008, 4, 435-447.

(41) Hockney, R.; Goel, S.; Eastwood, J. Quiet high resolution computer models of a plasma. J. Comput. Phys. 1974, 14, 148-158.

(42) Bussi, G.; Donadio, D.; Parrinello, M. Canonical sampling through velocity rescaling. J. Chem. Phys. 2007, 126, 014101.

(43) Berendsen, H. J. C.; Postma, J. P. M.; van Gunsteren, W. F.; DiNola, A.; Haak, J. R. Molecular dynamics with coupling to an external bath. J. Chem. Phys. 1984, 81, 3684-3690.
(44) Parrinello, M.; Rahman, A. Polymorphic transitions in single crystals: A new molecular dynamics method. J. Appl. Phys. 1981, 52, $7182-7190$.

(45) Martínez, L.; Andrade, R.; Birgin, E. G.; Martínez, J. M. PACKMOL: A package for building initial configurations for molecular dynamics simulations. J. Comput. Chem. 2009, 30, 21572164.

(46) Hoshen, J.; Kopelman, R. Percolation and cluster distribution. I. Cluster multiple labeling technique and critical concentration algorithm. Phys. Rev. B 1976, 14, 3438-3445.

(47) Essmann, U.; Perera, L.; Berkowitz, M. L.; Darden, T.; Lee, H.; Pedersen, L. G. A smooth particle mesh Ewald method. J. Chem. Phys. 1995, 103, 8577-8593.

(48) Yesylevskyy, S. O.; Schäfer, L. V.; Sengupta, D.; Marrink, S. J. Polarizable Water Model for the Coarse Grained MARTINI Force Field. PLoS Comput. Biol. 2010, 6, e1000810.

(49) Wu, R.; Deng, M.; Kong, B.; Yang, X. Coarse Grained Molecular Dynamics Simulation of Ammonium Surfactant Self Assemblies: Micelles and Vesicles. J. Phys. Chem. B 2009, 113, 15010-15016.

(50) Jorge, M. Molecular Dynamics Simulation of Self Assembly of $\mathrm{n}$ Decyltrimethylammonium Bromide Micelles. Langmuir 2008, 24, 5714-5725.

(51) Atik, S. C. P. S.; Thomas, J. K. Photoprocesses in cationic microemulsion systems. J. Am. Chem. Soc. 1981, 103, 4367-4371.

(52) Berr, S.; Jones, R. R. M.; Johnson, J. S. Effect of counterion on the size and charge of alkyltrimethylammonium halide micelles as a function of chain length and concentration as determined by small angle neutron scattering. J. Phys. Chem. 1992, 96, 5611-5614.

(53) Ekwall, P.; Mandell, L.; Solyom, P. The aqueous cetyl trimethylammonium bromide solutions. J. Colloid Interface Sci. 1971, $35,519-528$.

(54) Hansson, P.; Jönsson, B.; Ström, C.; Söderman, O. Determination of Micellar Aggregation Numbers in Dilute Surfactant Systems with the Fluorescence Quenching Method. J. Phys. Chem. B 2000, 104, 3496-3506.

(55) Lianos, P.; Zana, R. Fluorescence probe studies of the effect of concentration on the state of aggregation of surfactants in aqueous solution. J. Colloid Interface Sci. 1981, 84, 100-107.

(56) Quirion, F.; Magid, L. J. Growth and counterion binding of cetyltrimethylammonium bromide aggregates at 25.degree.C: a neutron and light scattering study. J. Phys. Chem. 1986, 90, 54355441.

(57) Ribeiro, A. C. F.; Lobo, V. M. M.; Valente, A. J. M.; Azevedo, E. F. G.; Miguel, M. G.; Burrows, H. Transport properties of alkyltrimethylammonium bromide surfactants in aqueous solutions. Colloid Polym. Sci. 2004, 283, 277-283.

(58) Clausen, T. M.; Vinson, P. K.; Minter, J. R.; Davis, H. T.; Talmon, Y.; Miller, W. G. Viscoelastic micellar solutions: microscopy and rheology. J. Phys. Chem. 1992, 96, 474-484.

(59) Olsson, U.; Soederman, O.; Guering, P. Characterization of micellar aggregates in viscoelastic surfactant solutions. A nuclear magnetic resonance and light scattering study. J. Phys. Chem. 1986, 90, $5223-5232$.

(60) Rao, U. R. K.; Manohar, C.; Valaulikar, B. S.; Iyer, R. M. Micellar chain model for the origin of the visoelasticity in dilute surfactant solutions. J. Phys. Chem. 1987, 91, 3286-3291.

(61) Brinker, C. J. Porous inorganic materials. Curr. Opin. Solid State Mater. Sci. 1996, 1, 798-805.

(62) Šefćík, J.; McCormick, A. V. Thermochemistry of aqueous silicate solution precursors to ceramics. AIChE J. 1997, 43, 27732784.

(63) Chen, C. Y.; Burkett, S. L.; Li, H. X.; Davis, M. E. Studies on mesoporous materials II. Synthesis mechanism of MCM 41. Micro porous Mater. 1993, 2, 27-34.

(64) Lin, Z. Branched Worm like Micelles and Their Networks. Langmuir 1996, 12, 1729-1737.

(65) Dreiss, C. A. Wormlike micelles: where do we stand? Recent developments, linear rheology and scattering techniques. Soft Matter 2007, 3, 956-970. 
(66) Magid, L. J.; Han, Z.; Li, Z.; Butler, P. D. Tuning the Contour Lengths and Persistence Lengths of Cationic Micelles: The Role of Electrostatics and Specific Ion Binding. J. Phys. Chem. B 2000, 104, 6717-6727.

(67) Devreux, F.; Boilot, J. P.; Chaput, F.; Lecomte, A. Sol gel condensation of rapidly hydrolyzed silicon alkoxides: A joint ${ }^{29} \mathrm{Si}$ NMR and small angle x ray scattering study. Phys. Rev. A: At., Mol., Opt. Phys. 1990, 41, 6901-6909.

(68) Israelachvili, J. N. Intermolecular and Surface Forces; Academic Press: London, U.K., 1985.

(69) Chen, C. Y.; Li, H. X.; Davis, M. E. Studies on mesoporous materials. Microporous Mater. 1993, 2, 17-26.

(70) Kruk, M.; Jaroniec, M.; Sayari, A. Application of Large Pore MCM 41 Molecular Sieves To Improve Pore Size Analysis Using Nitrogen Adsorption Measurements. Langmuir 1997, 13, 6267-6273.

(71) Lee, J. W.; Shim, W. G.; Moon, H. Adsorption equilibrium and kinetics for capillary condensation of trichloroethylene on MCM 41 and MCM 48. Microporous Mesoporous Mater. 2004, 73, 109-119.

(72) Corma, A.; Moliner, M.; Diaz Cabanas, M. J.; Serna, P.; Femenia, B.; Primo, J.; Garcia, H. Biomimetic synthesis of micro porous and mesoporous materials at room temperature and neutral $\mathrm{pH}$, with application in electronics, controlled release of chemicals, and catalysis. New J. Chem. 2008, 32, 1338-1345.

(73) Sayari, A. Recent advances and new horizons in zeolite science and technology. In Studies in Surface Science and Cataysis; Chon, H., Woo, S. I., Park, S. E., Eds.; Elsevier, 1996; Vol. 102, pp 1-46.

(74) Kruk, M.; Jaroniec, M.; Sakamoto, Y.; Terasaki, O.; Ryoo, R.; Ko, C. H. Determination of Pore Size and Pore Wall Structure of MCM 41 by Using Nitrogen Adsorption, Transmission Electron Microscopy, and X ray Diffraction. J. Phys. Chem. B 2000, 104, 292301.

(75) Chen, H.; Wang, Y. Preparation of MCM 41 with high thermal stability and complementary textural porosity. Ceram. Int. 2002, 28, 541-547.

(76) Schulz Ekloff, G.; Rathouský, J.; Zukal, A. Mesoporous silica with controlled porous structure and regular morphology. Int. J. Inorg. Mater. 1999, 1, 97-102.

(77) Firouzi, A.; Kumar, D.; Bull, L. M.; Besier, T.; Sieger, P.; Huo, Q.; Walker, S. A.; Zasadzinski, J. A.; Glinka, C.; Nicol, J.; Margolese, D.; Stucky, G. D.; Chmelka, B. F. Cooperative organization of inorganic surfactant and biomimetic assemblies. Science 1995, 267, 1138.

(78) Knight, C. T. Are zeolite secondary building units really red herrings? Zeolites 1990, 10, 140-144.

(79) Mora Fonz, M. J.; Catlow, C. R. A.; Lewis, D. W. Oligomerization and Cyclization Processes in the Nucleation of Microporous Silicas. Angew. Chem., Int. Ed. 2005, 44, 3082-3086.

(80) Malani, A.; Auerbach, S. M.; Monson, P. A. Probing the Mechanism of Silica Polymerization at Ambient Temperatures using Monte Carlo Simulations. J. Phys. Chem. Lett. 2010, 1, 3219-3224.

(81) Malani, A.; Auerbach, S. M.; Monson, P. A. Monte Carlo Simulations of Silica Polymerization and Network Formation. J. Phys. Chem. C 2011, 115, 15988-16000. 